\title{
Presenting Lengthy Problem-Solving Techniques With PowerPoint
}

\author{
Lynn R. Thomas, (E-mail: lynnthom@ksu.edu), Kansas State University
}

\begin{abstract}
This article illustrates the use of PowerPoint presentations in teaching problem-solving techniques where more than a simple "uncovering" of a line-by-line solution is desirable. A major hurdle in using PowerPoint to present solutions to accounting problems is getting copious amounts of material on the screen while keeping the font size large enough to be seen by students in the back of the room. Several techniques for overcoming this hurdle are discussed and illustrated. Two exercises involving the statement of cash flows have been chosen as examples. Lastly, the URL of the author's website is provided so that interested readers may download approximately 100 PowerPoint slides if they wish to incorporate or modify these presentations for use in their own classroom.
\end{abstract}

\section{Introduction}

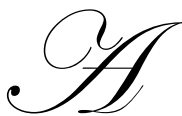

lthough multi-media presentations in the classroom have drawn a large amount of interest over the past several years, one can argue that accounting textbook authors and/or publishers have failed to provide much in the way of multimedia presentation materials beyond the standard PowerPoint outlines of their chapters and transparencies of homework solutions. Perhaps one of the reasons for this paucity of presentation materials is the inherent difficulty in getting a substantial amount of material on a PowerPoint slide while maintaining a readable font size. This article suggests some tips for overcoming this dilemma, while illustrating the use of PowerPoint presentations in teaching problem solving techniques where more than a simple "uncovering" of a line-by-line solution is desirable. The goals of these PowerPoint presentations are to: 1) proceed through a step-by-step problem solving procedure without divulging any of the solution prior to its concomitant discussion; 2) maintain as much of the solution as possible on the slide at all times (the big picture); 3) use a font size that is large enough to be read by students in the back of a normal classroom (say, seven rows of tables) with a normal sized projection screen (seven feet wide and five feet tall); and 4) avoid having to use a chalkboard or other ancillary device to supplement the presentation.

The topics chosen for illustration are the preparation of a statement of cash flows (SCF) under the direct method as well as the worksheet approach for preparing an indirect method statement.

The URL to the author's website is provided (http://info.cba.ksu.edu/Thomas/Public/ KWWEx23_13_21.ppt/) so that interested readers may download the 44 slides for the non-worksheet exercise and/or the 43 slides for the worksheet exercise. These slides were designed as a "talk-through" presentation as opposed to asynchronous use by students. Therefore, examples of lecture commentary are also discussed herein.

\section{Preparation Of A Statement Of Cash Flows Under The Direct Method}

The first illustration is a medium-level-of-difficulty exercise whose requirement is to prepare a statement of cash flows under the direct method. The exercise is not reproduced here but can be found as Exercise 23-13 on page 1251 of the Eleventh Edition ${ }^{1}$ of Intermediate Accounting by Kieso, Weygandt, and Warfield, a leading textbook in

\footnotetext{
${ }^{1}$ This exercise can also be found in the Tenth Edition as E24-15.
} 
its field. This is a typical SCF exercise in that comparative balance sheets and an income statement are presented, along with pieces of additional information concerning dividends, a sale of equipment, etc.

The basic technique utilized in this first example is to keep the outline of the desired SCF on the screen at all times, filling in that outline as one goes through the problem. An additional technique is to rotationally perform the computations in a blank area of the slide while instructing the students to place the computations off to the side on their hard copy ${ }^{2}$. In order to speed things along (this exercise can easily consume a fifty-minute class period) and to avoid confusion, the author usually provides the students with the handout shown in Figure $1^{3}$.

\section{Figure 1}

\begin{tabular}{|c|c|} 
Statement of Cash Flows & (1) \\
F23-13 $\begin{array}{c}\text { For the Year ended December 31, 2005 } \\
\text { - Cash flows from operating activities: } \\
\text { Cash received from customers (1) }\end{array}$ & \\
- Cash flows from investing activities: & \\
Cash flows from financing activities: & \\
\end{tabular}

Please note the following about this handout: 1) the font size is smaller than on the upcoming slides, since the students do not have to read the handout from a distance; 2) it contains the formal heading of the statement, whereas the slides do not, due to space limitations; and 3) the location for the placement of their first computation is indicated, in order to stress that they should not place those computations on the face of the SCF, as they will appear on the slides.

The presentation consists of "building" each subsequent slide after asking the students to describe the next step (the procedures have been covered previously in a lecture). For example, the first line in the operating activities section is described as "Cash received from customers." After receiving the students' responses to the question of the calculation method, the next slide (Figure 2) is presented, which shows the appropriate computation(s). The students are reminded once again to not write the computation where it appears on the slide but rather, off to the right side of their handout (where the "(1)" appears).

\footnotetext{
${ }^{2}$ While more sophisticated methods are possible, this procedure of using a blank space was chosen because of its simplicity of use and the fact that none of the rest of the slide is covered and rendered unreadable.

${ }^{3}$ This handout was also prepared in PowerPoint and is available for downloading along with a complete set of the slides.
} 
Figure 2

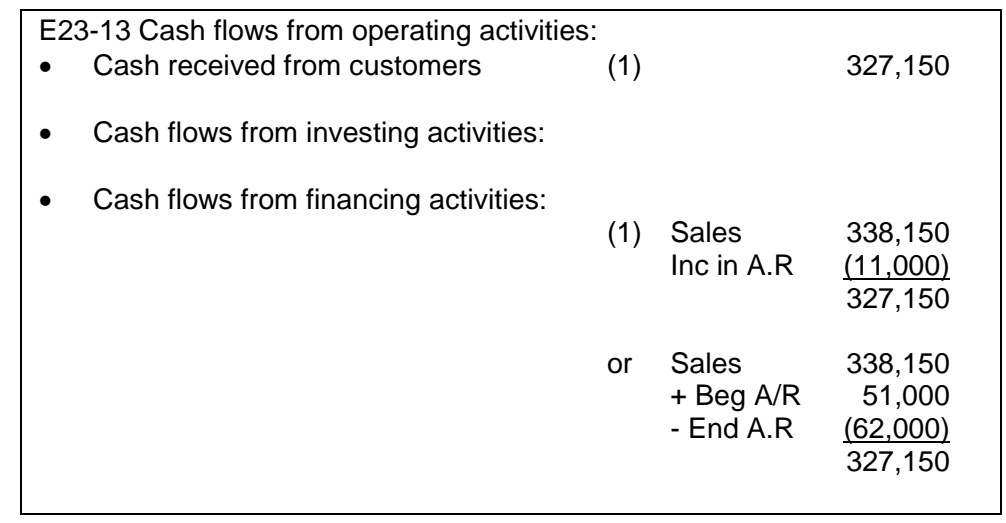

After looking in the exercise for evidence of other operating cash inflows and finding none, students are instructed to make a note to themselves in the left margin of their solution (handout) that any other cash inflows from operating activities should appear immediately following "Cash received from customers."

The slide show solution continues one step at a time, retaining the lines that make up the formal statement of cash flows, but deleting the computation, so that the next computation can utilize the same available blank space. Some of the computations are more complicated, and T-Accounts are "built" for analytical purposes in order to compute, for example, the amount of depreciation expense. Figure 3 depicts the last of four slides analyzing the accumulated depreciation account in order to compute the $\$ 24,000$ of depreciation expense that needs to be deducted from total operating expenses as part of the computation of "cash paid for operating expenses."

\section{Figure 3}

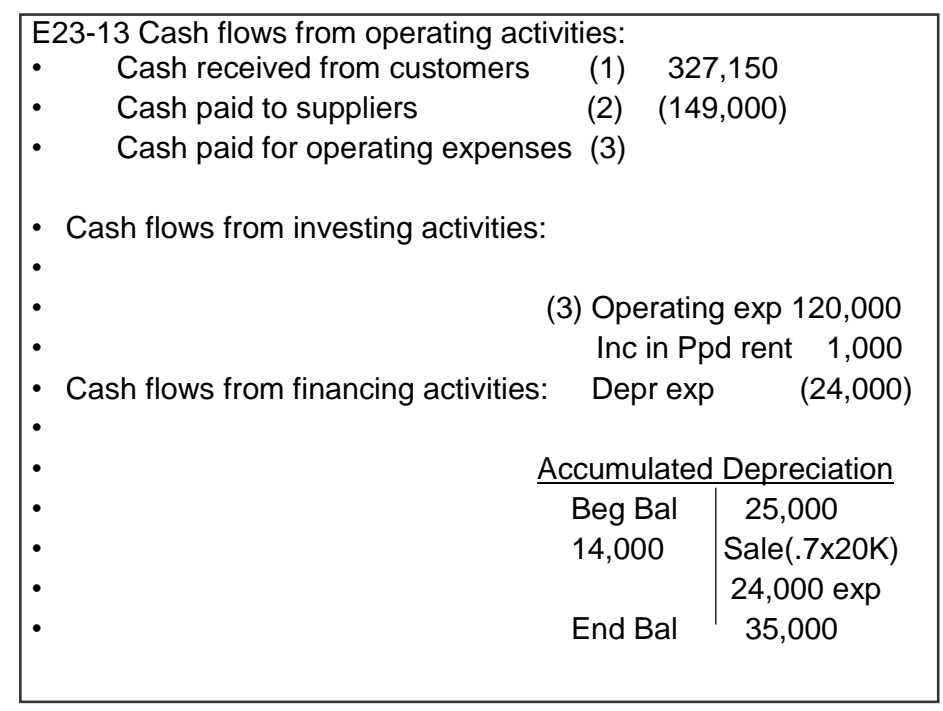

As the solution continues and more lines of the statement are completed, the first few lines are "scrolled" off the top of the slide in order to keep the font size large enough to be read from the back of the classroom. Figure 4 illustrates the final slide, but does not contain the entire statement, the top having been "scrolled" off the slide ${ }^{4}$.

\footnotetext{
${ }^{4}$ Although not illustrated, the set of downloadable slides also contains 11 slides for E23-14, requiring the preparation of the SCF under the indirect method for the same set of data.
} 
Figure 4

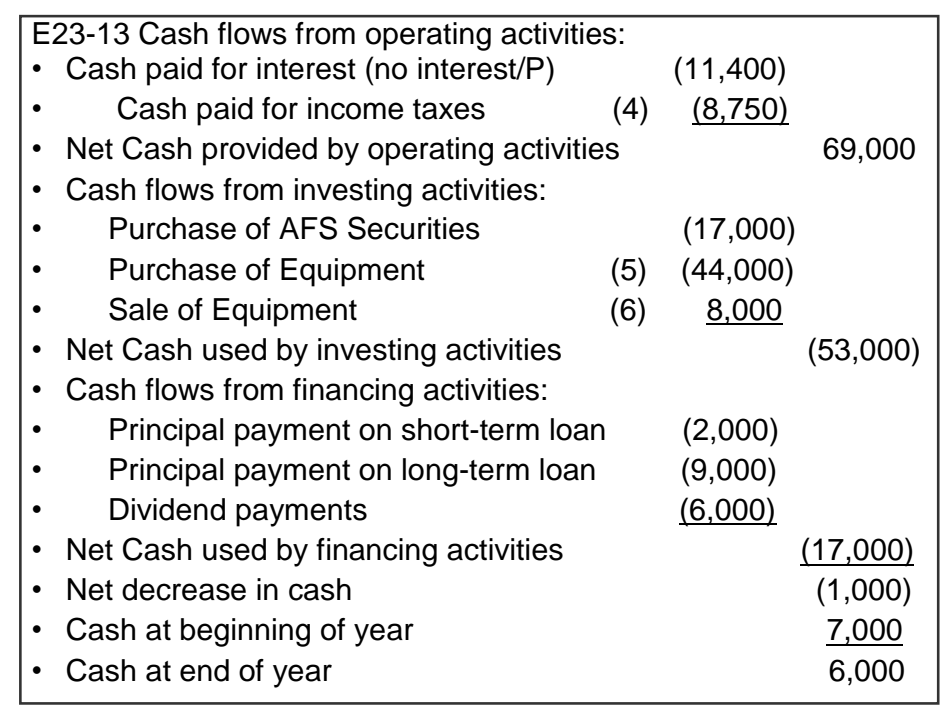

\section{Utilization Of A Worksheet To Prepare An Informal SCF Under The Indirect Method}

The second illustration is an exercise requiring the preparation of a worksheet SCF for a simplistic scenario (e.g., no sales of assets). Again, the exercise is not reproduced here, but can be found as Exercise 23-21 on page 1254 of the Eleventh Edition ${ }^{5}$ of Intermediate Accounting by Kieso, Weygandt, and Warfield. This exercise gives comparative balance sheet information in an unclassified (post-adjusted trial balance) format.

The basic technique utilized in this second example is to keep the bottom portion of the worksheet on the screen at all times (until "scroll-off" is necessitated), but sequentially rotate the various ledger accounts on the top part of the worksheet so that only one account is shown at a time ${ }^{6}$. Similar to the first illustration, in order to speed things up and minimize confusion, the author usually provides the class with the handout depicted in Figure 5.

Figure 5

\begin{tabular}{|c|c|c|c|c|}
\hline Account & 12-31-04 Balance & Debit & Credit & 12-31-05 Balance \\
\hline Cash & & & & \\
Retained Earnings & & & & \\
\hline OPERATING ACTIV & & & & \\
INVESTING ACTIV & & & & \\
FINANCING ACTIV & & & & \\
\hline
\end{tabular}

\footnotetext{
${ }^{5}$ This exercise can also be found in the Tenth Edition as E24-23.

${ }^{6}$ If several accounts are related to a single line on the SCF, all of those accounts are shown on the same slide.
} 
Net income is not given in this exercise, but we are told that dividends of $\$ 15,000$ were declared and paid in 2005. Since the indirect method SCF starts with net income, the retained earnings account needs to be "reconstructed" first, in order to compute the amount of said income. The first slide is depicted in Figure 6.

Figure 6

\begin{tabular}{|l|l|}
\hline Retained Earnings 51,500 & 73,400 \\
- OPERATING ACTIVITIES: & \\
- Net Income & $?$ \\
- INVESTING ACTIVITIES: & \\
- FINANCING ACTIVITIES: & \\
\hline
\end{tabular}

Students need to be apprised of what they are looking at on the slide compared to their handout. They also need to be reminded that the columns have been reversed compared to the comparative balance sheets because it is easier for most people to think from left to right across the page. Dividends would have been debited to retained earnings by the company and we need to reconstruct that debit on the top part of the worksheet, forcing out a credit in the "financing activities" section on the bottom portion of the worksheet (the informal SCF). This debiting and crediting is done one step at a time over three slides so that the students can be walked slowly (or quizzed) through the thought process. The balancing credit to retained earnings is the amount of net income, and is illustrated in Figure 7.

Once the analysis of retained earnings has been completed the illustrated solution goes back up to the second line of the balance sheet and proceeds to reconstruct the entries (if any) for the remainder of the accounts. Accounts receivable and the allowance for doubtful accounts are analyzed at the same time, as illustrated in Figure 8. (Figure 8 is the final of a three-step "build" so that students can be asked about the debit/credit reconstruction).

After the remaining accounts are reconstructed and offsetting entries made in the SCF, the presentation culminates with a slide that depicts the total of the debit and credit columns. Students are reminded that this "informal" SCF would then be used to create a "formal" SCF. 
Figure 7

Figure 8

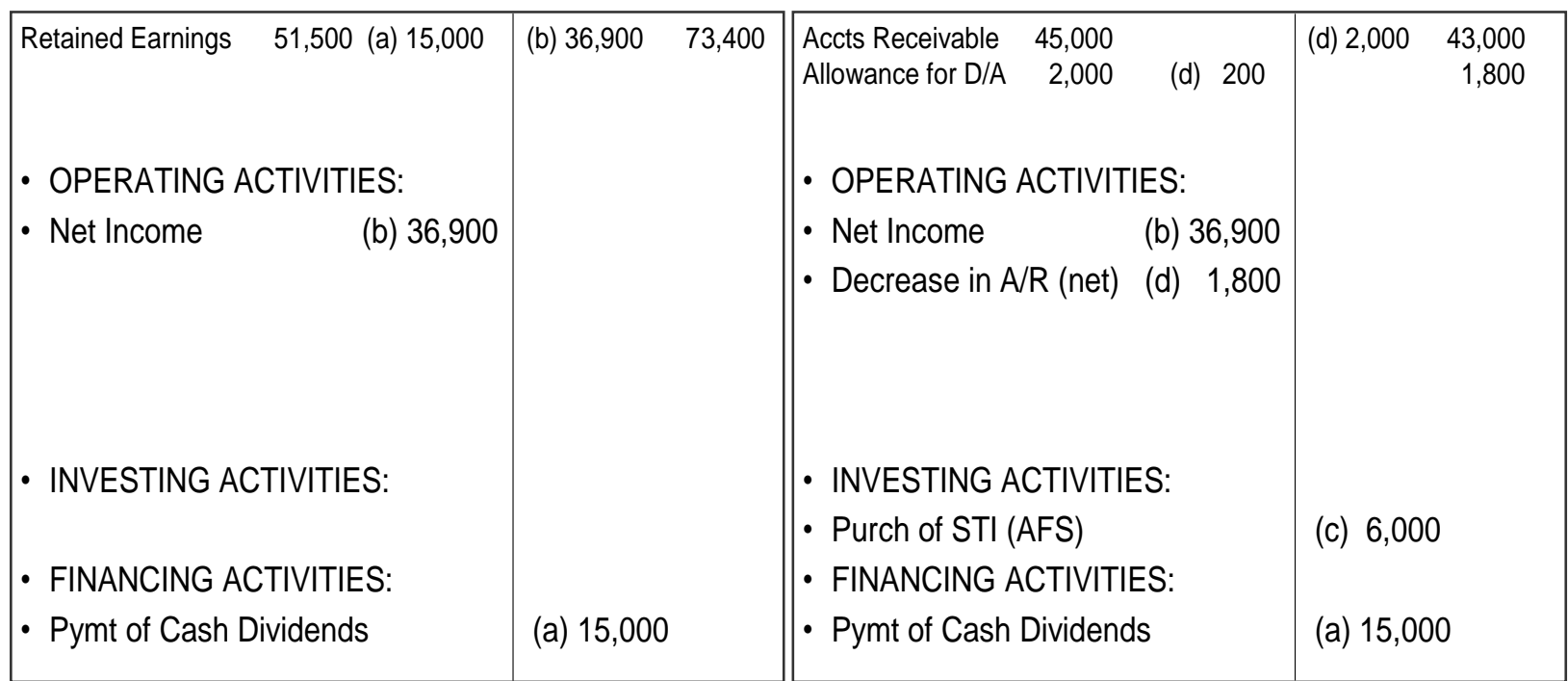

\section{Summary And Additional Tips}

This article illustrates certain techniques for using PowerPoint slides to present lengthy solutions to two exercises requiring the preparation of statements of cash flows. These techniques include the rotation of computations on and then off the slides and "scrolling" off the top portion of the statement when it becomes too lengthy. These techniques are accomplished without: 1) divulging any of the solution prior to its simultaneous discussion; 2) removing the basic solution from the slide; 3 ) reducing the font size so that it is not readable from the back of the room; or 4) having to use a chalkboard or other ancillary device to supplement the presentation.

Since slide shows similar to the two illustrated above frequently contain more than 40 individual slides, the correction of errors (or shortcomings) is very time consuming. Therefore, in addition to an admonition to work carefully, the following suggestions are made. First, minimize the use of exercise or problem numbers, company names, and dates on the slides (except an introductory slide). Textbook authors like to change dates, but if they don't change any numbers, you might not have to redo all of your slides when that new edition comes out.

Second, spend a little extra time planning ahead and making sure that you have everything on your first slides exactly the way you want it to appear on your last slides. For example, put dollar signs in the slide if applicable, and allow enough space for larger numbers or longer account titles if they are going to occur later on.

Additionally, consider moving the margins on all four sides of your PowerPoint master slide clear to the edge of the slide (as was done in the above illustrations). This allows for a significant increase in the number of letters and digits that will fit on the slide. Be sure to use a true font, such as Arial or Times New Roman. Use of a Universal font will result in columns going berserk when you shut off your computer or move from one computer to another.

Lastly, the author's URL (http://info.cba.ksu.edu/Thomas/Public/KWWEx23_13_21.ppt/) is provided so that readers can view the entire set of slides and/or download them for their own use. 